\title{
Fatty necrosis of falciform ligament due to torsion mimics falciform ligament cyst on MRI
}

\author{
İlknur Özdeniz', Ali Önder Devay², Ediz Tevfik Özgan² \\ ${ }^{1}$ Department of Radiology, Bursa State Hospital, Bursa, Turkey \\ ${ }^{2}$ Department of General Surgery, Bursa State Hospital, Bursa, Turkey
}

DOI: $10.18621 /$ eurj.347535

\begin{abstract}
The falciform ligament which is a broad and thin peritoneal ligament, divides the left and right subphrenic compartments. Some pathologies of this ligament can be a rare cause of acute abdomen. Mostly its pathologies present a challenge for diagnose. In a case who was admitted with acute abdomen, we determined a fatty necrosis of the falciform ligament during surgery. This rare primary pathology of the falciform ligament mimics falciform ligament cyst on MRI, which is another rare pathology.
\end{abstract}

Keywords: Falciform ligament, fatty necrosis, cyst, MRI

Received: October 28, 2017; Accepted: December 26, 2017; Published Online: March 17, 2018

$\mathrm{T}$ he falciform ligament is a double layer of peritoneum that goes from the upper and anterior surfaces of the liver to the lower surface of the diaphragm and the back of the linea alba. Its line of attachment divides the liver into left and right lobes [1]. It attaches the liver to the remnants of the umbilical veins. It contains the ligamentum teres, obliterated umbilical vein and paraumbilical veins. The falciform ligament artery mostly arises from middle or left hepatic artery [2]. Furthermore, falciform ligament-related conditions are very rare.

In this paper, we present a case with falciform ligament necrosis undergoing surgery, that it was clinically thought as acute cholecystitis. It was diagnosed by surgery and histologic findings. This pathology confirmed with unusual MRI feature mimics falciform ligament cyst and disappeared after 1-month on MRI.

\section{CASE PRESENTATION}

A 46-year-old female patient was admitted with the complaints of pain in the right upper quadrant. The patient's history revealed an abdominal pain for 6 hours with an increasing severity, as well as nausea and vomiting. Physical examination suggested acute cholecystitis with a systolic blood pressure 160/85 $\mathrm{mmHg}$, pulse rate of $97 \mathrm{bpm}$, and body temperature of $37.4^{\circ} \mathrm{C}$. Laboratory test results showed a leukocyte count of $15.000 / \mathrm{mm} 3$ (normal range: $4.000-$ $10.000 / \mathrm{mm} 3)$. Serum chemistry tests and electrolyte measurements did not indicate any pathology related to acute abdomen (ALT $8 \mathrm{U} / \mathrm{L}$, AST $10 \mathrm{U} / \mathrm{L}$, amylase $52 \mathrm{U} / \mathrm{L}$, total bilirubin $1,1 \mathrm{mg} / \mathrm{dL}$ ).

Abdominal ultrasonography was requested because of suspected acute cholecystitis and demonstrated gallbladder with bile stone. However its 


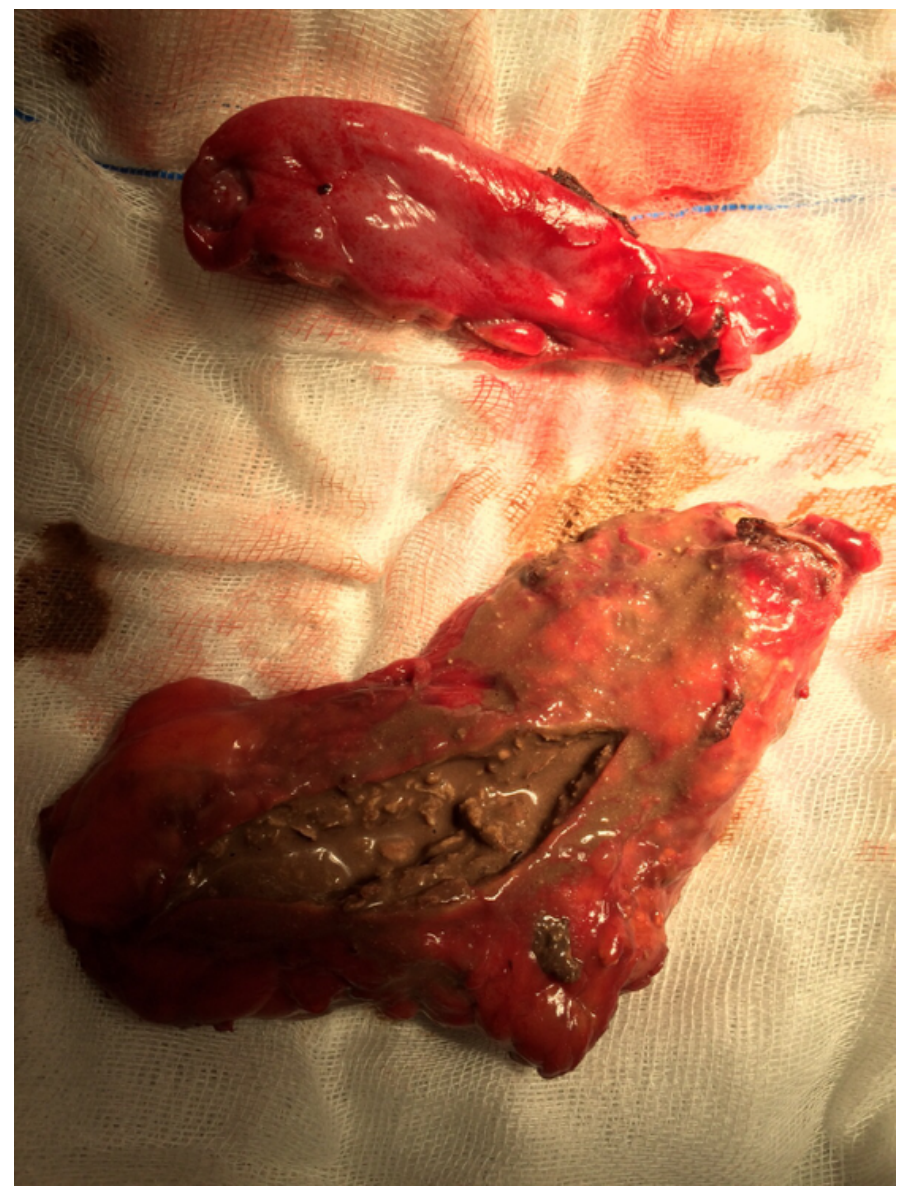

Figure 1. Macroscopic view of excised cystic structure and gallbladder

wall thickness was normal without edema. There was no heterogeneity localized in the falciform ligament. It's not completely suitable for the diagnosis of acute cholecystitis. Because the clinical condition of the patient supports this pathology, the patient accepted as acute cholecystitis and she underwent the surgery. In the operation, the gallbladder was resected but on the falciform ligament location there was a cysticliquefied structure without any normal falciform ligament portions (Figure 1). No pathology responsible for falciform ligament necrosis was observed. This cystic-necrotic structure could not completely resected. Since cystic necrotic structure was seen on falciform ligament location, it's taught that this structure can be evaluated better on MRI. So MRI was performed on the 4th day of the operation. Also due to the emergency conditions of patient before the operation, CT/MRI could not be done.

Postoperative 4th day, on MRI there was a cystic remnant on the falciform ligament location (Figures 2, 3, 4 and 5). The histopathological examination of

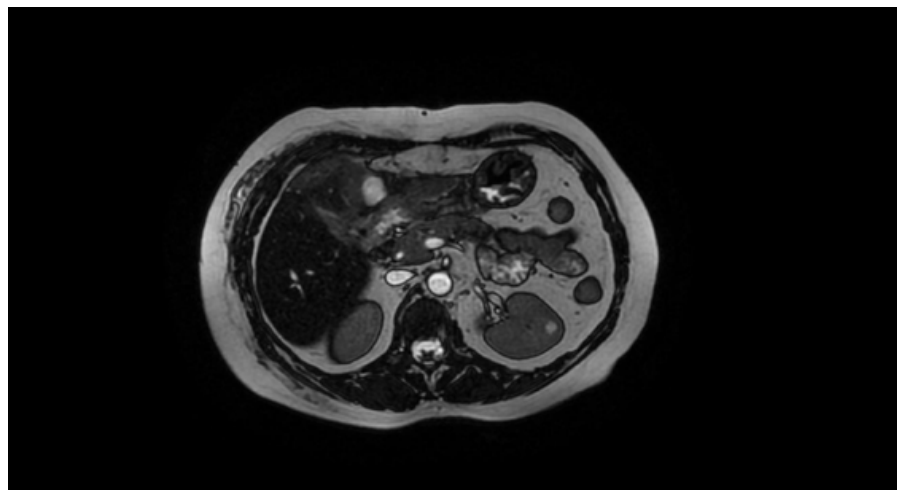

Figure 2. Postoperative 4th day, axial FIESTA MRI scan shows the remnant of cystic structure (This can also be post-surgical fluid collection, but in this case the most important cause that lead us to think as falciform ligament necrosis is the correlation with surgical findings. We knew that some part of the necrosed falciform ligament is resected by surgery).

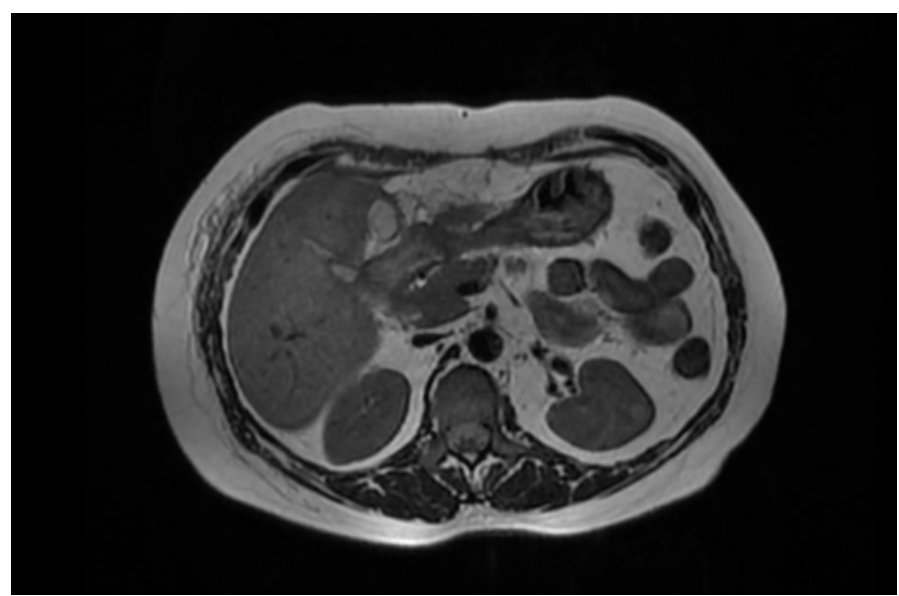

Figure 3. The same structure is hyperintense on axial T2 weighted image.

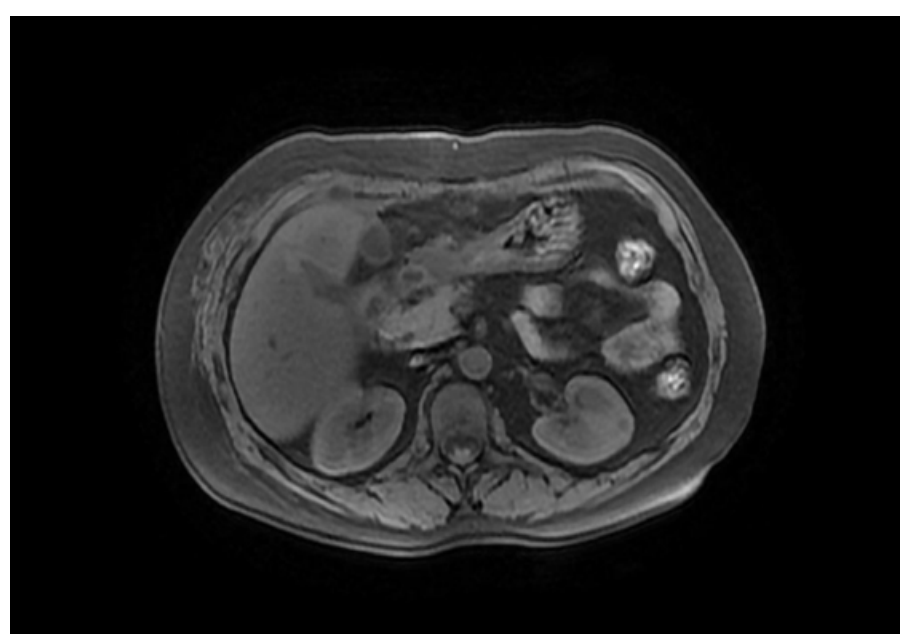

Figure 4. It is hypointense on axial T1 FSPGR fat saturated image. 


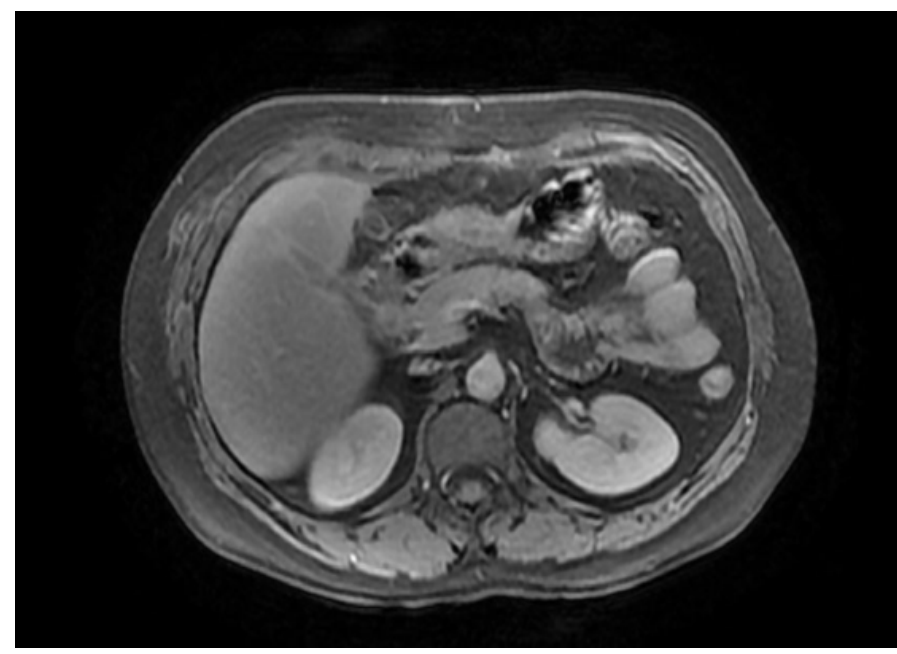

Figure 5. It is not enhanced on post-gadolinium axial T1 FSPGR fat saturated image.

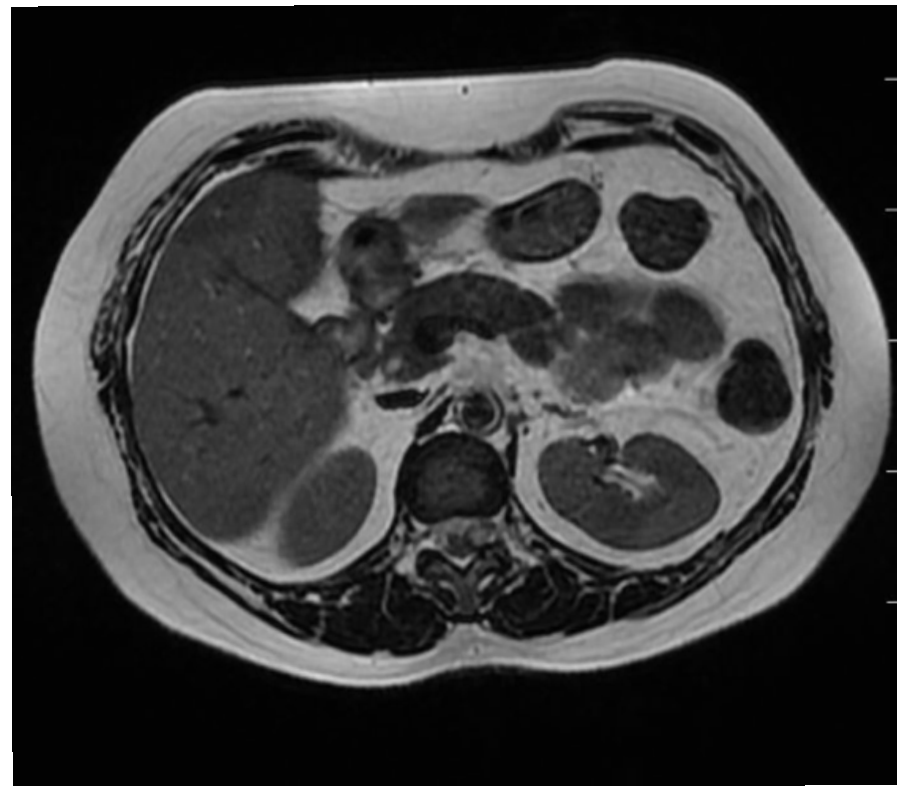

Figure 6. Postoperative 1st month the structure disappeared on axial T2 image.

surgical specimen revealed a falciform ligament fatty necrosis with liquefaction. One month after surgery, cyst on the falciform ligament location was disappeared (Figures 6 and 7).

\section{DISCUSSION}

Although the anatomical structure and variations of the falsiform ligament are well known, the associated pathologies are still unclear. The congenital

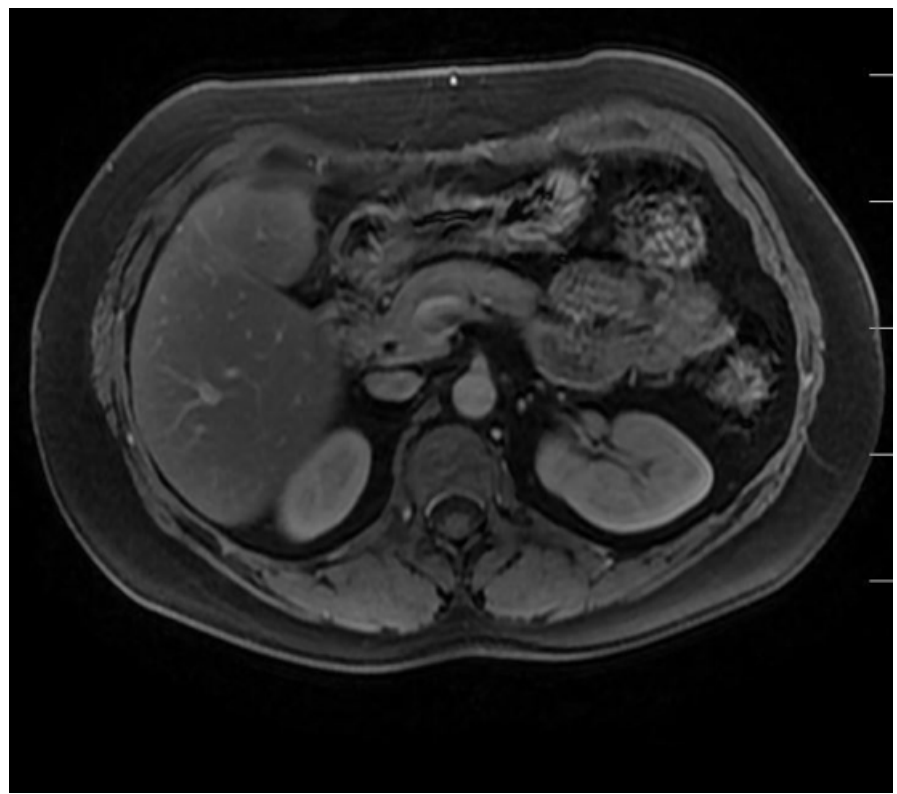

Figure 7. Postoperative 1st month post-gadolinium axial T1 FSPGR fat saturated image (this image is changed due to reviews).

pathologies including derivation and partial ligament defects are the best known anomalies of the falsiform ligament. The most common pathologies of this ligament are abnormal vascularity due to portal hypertension, tumors and ligament cysts [3]. There are still difficulties in diagnose of falciform ligament necrosis came with right upper quadrant pain as in our case.

The falciform ligament artery which arises from the left or middle hepatic artery, runs down the anterior surface of the liver, and reaches the umbilical region. Venous drainage flows directly into the paraumbilical vein and portal vein, while lymphatic drainage flows directly into the retroperitoneum [2]. Falciform ligament necrosis may likely develop, if the embolization of such a narrow artery or collateral venous flow fails due to vein thrombosis. Necrosis due to an occlusion of arterial supply is a rare primary falciform ligament disease [3].

The clinical picture and laboratory values are often nonspecific and may point wrongly towards diagnosis of biliary colic or cholecystitis as in our patient. Although very rare, radiologists should know this fatty necrosis of the falciform ligament, in order to avoid diagnostic error and optimize patient management. It was reported that the contrast-enhanced abdominal computed tomography is the gold standard for 
diagnosis of intraperitoneal fat necrosis as well as the follow-up of the disease [4]. However, there are few recent data on MRI in the literature about falciform ligament pathologies. The liquefaction of the falciform ligament due to fatty necrosis can mimics falciform ligament cysts on MRI as in our case.

Although there have been defined falciform ligament cysts in literature, most recently published in 2009 [5]. Primary cysts include congenital developmental defects of mesenteric origin; secondary cysts include infectious origins (echinococcal cyst and abscess), traumatic causes (liquefaction of a hematoma and bile extravasation), and neoplasms with cystic degeneration [5]. Radiologic features should be evaluated with clinical and histologic findings to distinguish ligament cysts from necrosis.

\section{CONCLUSION}

In conclusion, falciform ligament necrosis which is an extremely rare cause of acute abdomen can have different imaging features. It can mimic other pathologies of falciform ligament especially on imaging modalities and also during surgery. It is important to consider clinical, histologic and radiologic findings all together.

\section{Informed consent}

Written informed consent was obtained from the patient for the publication of this case report.

\section{Conflict of interest}

The authors declared that there are no potential conflicts of interest with respect to the research, authorship, and/or publication of this article.

\section{REFERENCES}

[1] Maccallum C, Eaton S, Chubb D, Franzi S. Torsion of fatty appendage of falciform ligament: acute abdomen in a child. Case Rep Radiol 2015;2015:293491.

[2] Ibukuro K, Fukuda H, Tobe K, Akita K, Takeguchi T. The vascular anatomy of the ligaments of the liver: gross anatomy, imaging and clinical applications. Br J Radiol 2016;89:20150925.

[3] Ozkecec1 ZT, Ozsoy M, Celep B, Bal A, Polat C. A rare cause of acute abdomen: an isolated falciform ligament necrosis. Case Rep Emerg Med 2014;2014:570751.

[4] Coulier B. Contribution of US and CT for diagnosis of intraperitoneal focal fat infarction (IFFI): a pictorial review. JBR-BTR 2010;93:17185.

[5] Patel A, Lefemine V, Ramanand BS. A rare case of a peritoneal cyst arising from the falciform ligament. Cases J 2009;2:134. 